\title{
La historia del concepto de desnaturalización de desastres
}

\author{
A História do conceito de Desnaturalização de Desastres \\ The History of the Disaster Denaturalization Concept
}

Virginia García-Acosta ${ }^{1}$

\section{Resumen}

GARCÍA-ACOSTA, V. La historia del concepto de desnaturalización de desastres.

Rev. C\&Trópico, v. 45, n. 2, p. 159-166, 2021. DOI: https://doi.org/10.33148/cetropi$\operatorname{cov} 45 n 2(2021) \operatorname{art} 9$

En el transcurso de presentar la historia de la formulación del concepto de desnaturalización, su importancia y relevancia para el momento actual en las ciencias sociales, son consideradas las importantes implicaciones del concepto de desnaturalización con los conceptos de construcción social de prevención y vulnerabilidades sociales asociadas a desastres. En este artículo, entonces, se destaca que las estrategias de adaptación exitosas se construyen a partir de las estructuras organizativas de las comunidades. En el caso de la pandemia de COVID-19, también se considera la importancia de su desnaturalización biológica. Este texto concluye que tenemos mucho que aprender en el campo de los desastres, la desnaturalización, la resistencia que surgió y las movilizaciones en curso que están por venir.

Palabras clave: Desnaturalización de desastres. Construcción social de prevención. Vulnerabilidades sociales. COVID-19.

\section{Resumo}

GARCÍA-ACOSTA, V. A História do conceito de Desnaturalização de Desastres. Rev. CઐTrópico, v. 45, n. 2, p. 159-166, 2021. DOI: https://doi.org/10.33148/cetropicov45n2(2021)art9

No percurso da apresentação da história da formulação do conceito de desnaturalização, sua importância e relevância para o momento atual nas ciências sociais, são consideradas as importantes implicações do conceito de desnaturalização com os conceitos de construção social da prevenção e vulnerabilidades sociais associadas aos desastres. Neste artigo, então, destaca-se que estratégias adaptativas de sucesso são construídas a partir das estruturas organizacionais de comunidades. No caso da pandemia COVID-19, também é considerada a importância de sua desnaturalização biológica. Conclui-se com este texto que temos muito a aprender no campo

1 Antropóloga social e historiadora mexicana. Profesora-Investigadora del CIESAS desde 1973. Sus áreas de especialidad son antropología e historia del riesgo y de los desastres en México y América Latina. Es miembro de número de la Academia Mexicana de la Historia (2013), de la Academia Mexicana de Ciencias (1987) y del Sistema Nacional de Investigadores desde 1987. E-mail: vgarciaa@ciesas.edu.mx Orcid: https://orcid.org/0000-0001-7398-5478 
dos desastres, da desnaturalização, das resistências que surgiram e das mobilizações em andamento e que ainda estão por vir.

Palavras-chave: Desnaturalização de desastres. Construção social da prevenção. Vulnerabilidades sociais. COVID-19.

\section{Abstract \\ GARCÍA-ACOSTA, V. The History of the Disaster Denaturalization Concept. Rev. CひTró- pico, v. 45, n. 2, p. 159-166, 2021. DOI: https://doi.org/10.33148/cetropicov45n2(2021)art9}

In order to present the history of the formulation of the concept of denaturalization, its importance and relevance for the current moment in the social sciences, the important implications of the concept of denaturalization with the concepts of social construction of prevention and social vulnerabilities associated with disasters are considered. In this article, therefore, it is highlighted that successful adaptive strategies are built from the organizational structures of communities. In the case of the COVID-19 pandemic, the importance of its biological denaturalization is also considered. This text concludes that we have a lot to learn in the field of disasters, denaturalization, the resistance that arose and the ongoing mobilizations that are yet to come.

Keywords: Denaturalization of disasters. Social construction of prevention. Social vulnerabilities. COVID-19.

\section{Desnaturalización y movilización comunitaria}

Como les digo a mis estudiantes, siempre que planteamos un problema debemos tener claro qué estamos diciendo. Como señaló Gabriel García Márquez en el prólogo de sus Doce cuentos peregrinos: ¿por qué doce, por qué cuentos y por qué peregrinos?

Desnaturalizar los desastres, algo que ahora gritamos a los cuatro vientos considerando que se trata de un cambio de paradigma y que mientras se siga manteniendo la idea y sobre todo la premisa de que los desastres no son naturales, no avanzaremos un milímetro en la prevención y atención a los desastres como procesos asociados con amenazas naturales o biológicas. Aunque nos parezca algo novedoso, la idea de desnaturalizar los desastres viene de muy atrás. Y eso lo sabemos gracias a estudiarlos históricamente. Vayan algunos ejemplos.

Una de las publicaciones prístinas en este sentido data de hace ya casi medio siglo. Se trata del artículo titulado'Taking the naturalness out of natural disasters', escrito por tres autores que desde entonces no quitan el dedo del renglón: Phil O’Keefe, 
Ken Westgate y Ben Wisner, y que fue publicado, paradójicamente, en la reconocida Revista Nature (O'KEEFE et al., 1976). Y digo paradójicamente porque precisamente la tesis del artículo, a partir de información proveniente de la ocurrencia de sequías, deslizamientos de tierra, temblores, ciclones en espacios como Bangladesh, Nigeria, Nicaragua, Guatemala, Ecuador o Venezuela es, como su título lo dice, retirar el adjetivo de "natural" del concepto desastre:

Es el momento para planear de forma preventiva (precautionary planning), al considerar a la vulnerabilidad de la población como la causa real de un desastre, una vulnerabilidad que puede ser modificada por el hombre [...] Planear para la prevención debe empezar eliminando la concepción de naturalidad (naturalness) de los desastres naturales (O'KEEFE et al., 1976, p. 567)

Las iniciativas en el mismo sentido continuaron, particularmente derivadas de conocer de manera directa la situación prevaleciente ante la ocurrencia de desastres y en gran parte proveniente del ahora denominado sur global. Entre ellas se encuentran reuniones de discusión que dieron lugar a publicaciones emblemáticas, en espacios distantes en el planeta en la década de los ochenta del siglo pasado. Me refiero en particular a las organizadas, una por el geógrafo Kenneth Hewitt en la Wilfried Laurier University, y la segunda por la Comisión de Desarrollo Urbano y Regional de CLACSO y el Centro de Estudios de la Realidad Económica y Social (CERES). Las publicaciones resultantes son ahora clásicas (HEWITT, 1983; CAPUTO et al., 1985).

Por otro lado la fundación en 1992 de LA RED (Red de estudios sociales en prevención de desastres en América Latina) en Costa Rica, con su primera publicación titulada precisamente Los desastres NO son naturales (MASKREY, 1993). Por cierto que una narrativa más amplia sobre este recorrido se ha publicado en varios escritos míos (GARCÍA ACOSTA, 2018), de Allan Lavell (2005), de Andrew Maskrey, en suma, de los fundadores de LA RED, que tiene su página: www.desenredando.org.

La influencia de estas perspectivas escaló a espacios dentro de organismos internacionales, tal como lo muestra la antropóloga Sandrine Revet en su cuidadosa etnografía sobre la comunidad internacional sobre desastres, Disasterland, a partir de la cual evidencia que el tema se ha posicionado como un asunto de negociaciones y decisiones en la materia alrededor del mundo, a la par de otros como salud global (global health), desarrollo y pobreza, crisis y conflicto, medio ambiente, cambio climático (REVET, 2020, p. 3-5).

Para incorporar en ellas esas miradas, que en términos generales podríamos denominar "los desastres no son naturales", la participación directa e indirecta de miembros de LA RED en ellos fue decisiva. La edición original del llibro de la antropóloga Revet apareció en 2018 como Les coulisses du monde des catastrophes "naturelles" (Éditions de la Maison des sciences de l'homme, Paris).

El estudio y análisis cuidadoso de la ocurrencia de desastres, tanto en el pasado 
como en el presente, en diferentes regiones del planeta y en condiciones y contextos diversos, considerando la pluralidad de factores y elementos que intervienen en ello, así como la identificación de las causas determinantes ha mostrado la obligación de distinguir claramente entre las manifestaciones de la naturaleza y sus efectos e impactos. Estos últimos se relacionan directamente con la construcción social de riesgos que ha incrementado, de manera creciente, la vulnerabilidad. De ahí la necesidad ineludible de desvincular esa subordinación de los desastres exclusivamente a causas naturales.

Dos marcos espacio-temporales constituyen hitos en esa "desnaturalización" de los desastres y su asociación inevitable con la vulnerabilidad. Dos décadas y 10 mil kilómetros los separan: África en la década de los setenta y América Latina en la de los noventa del siglo pasado. Pero sobre esto hemos ya escrito en otros espacios.

Si los desastres no son naturales, al menos no como nos los quieren presentar todavía en la actualidad sobre todo los tomadores de decisiones a escala regional, nacional e internacional, entonces ¿qué son?

Tenemos que empezar por definir lo que es un desastre, quizás partiendo de lo que no es:

- No es sinónimo de fenómeno natural;

- No es atribuible a la naturaleza como agente activo;

- $\quad$ No es un evento que ocurre en un momento y tiempo específicos.

Los desastres son procesos que se construyen históricamente. vayamos por partes.

Dos conceptos y nociones directamente relacionados con ese proceso: que forman parte del tema general de este Seminario: vulnerabilidad y riesgo... Y estrechamente relacionado con ellos se encuentra el concepto de construcción social asociado con los riesgos., el cual ha demostrado su utilidad analítica cada vez con mayor fuerza entre los estudiosos de los desastres y los efectos que estos han tenido en la sociedad. A pesar de su beneficio manifiesto para abordar la temática vinculada a los desastres, se le han atribuido significados diversos, lo cual ha contribuido en algunos casos a confusiones en su utilización. Esto es natural que ocurra en la evolución de los planteamientos teóricos que se construyen en las ciencias para la interpretación de la realidad.

\section{Construcción social del riesgo}

Si seguimos parafraseando a García Márquez con aquello del prólogo de su libro, y nos preguntamos ¿Por qué doce, por qué cuentos y por qué peregrinos?, les cuento que cuando le pregunté a Sergio Portella si tenían interés en que me centrara en algún aspecto en particular, me respondió que procurara seguir la "ementa" de divulgação da mesa-redonda, que señala lo siguiente: "Vulnerabilidades e desigualdades, base do atual modelo de desenvolvimento. Como resistimos? Como enfrentamos? Como modificamos? Os estudiosos conhecedores das experiências de resistência na América Latina, na África, na Ásia e mesmo na Europa, traçarão um quadro, um 
mapa, de como essas resistências e redes vêm se conformando atualmente para que as mudanças climáticas, desnaturalizadas, sejam enfrentadas como uma discussão operacional e não uma impossibilidade política”.

En el título de este Seminario y de la Mesa Redonda a la que me invitaron se habla de movilización y de resistencias. Estos asuntos tienen que ver con otro concepto que hemos venido desarrollando a partir de la información histórica y contemporánea estudiada, a partir de rastrear documentos y hacer trabajo de campo antropológico. El concepto al que me refiero es el de la construcción social de la prevención.

Existen numerosos ejemplos de buenas prácticas de adaptación, de respuesta y reacción ante las amenazas naturales a lo largo de la historia. Pero no se han explorado sistemáticamente a través de investigación rigurosa, en la documentación adecuada y con métodos apropiados y ya probados.

Se trata de formas a partir de las cuales la sociedad construye contextos preventivos ante la recurrencia de amenazas que provocan cambios permanentes en la cultura material y organizativa de las comunidades afectadas. Se trata de acciones, prácticas, estrategias identificadas a partir de documentos históricos y trabajo de campo.

Las estrategias adaptativas exitosas se montan sobre estructuras organizativas de la comunidad, a partir de la evaluación de los acontecimientos por parte del grupo afectado. Ello requiere de un sistema de redes bien estructurado (capital social) que se traduce en una transmisión del conocimiento experimentado por las siguientes generaciones. Constituye entonces parte de lo que denominamos "Construcción social de la prevención de riesgo de desastre".

A pesar de que se trata de un tema que ya hemos explorado a escala histórica y etnográfica, aún es un tema virgen.

La realidad evidencia que es cada vez más urgente explorar a fondo los elementos constitutivos de la Construcción social de la prevención, tanto a escala local y regional. Con ello queremos decir, identificar las estrategias que las comunidades y grupos sociales han desarrollado (documentos, etnografía critica) y, con todo este bagaje. trabajar más intensamente en la gestión local del riesgo de desastre (GLRD).

Ahora, si podemos discutir temas como los que he tocado aquí, como los que tocaron quienes participaron en la I Mesa redonda hace un par de horas y los que lo harán en las Mesas que seguirán como parte de este Seminario, es porque hay un cúmulo de investigación, de investigación teórica y práctica, de investigación-acción, histórica y contemporánea, que nos ha permitido avanzar en la temática. Nos preguntamos entonces, ¿por qué en ocasiones parece que partimos de cero? Como es el caso de la pandemia que estamos viviendo a escala global. ¿Por qué partir de cero?

Uno de los muchos ejemplos es el del concepto de vulnerabilidad, que ha sido objeto de numerosas definiciones, desde perspectivas distintas y con usos muy disímiles. Sólo por detenernos en uno. James Mitchell (Rutgers, State University of New York), geógrafo, propuso desde 2004 y 2005, el concepto de "vulnerable functions", "funciones vulnerables", que fue poco atendido. Lo retoma en una publicación reciente, con algunas modificaciones, precisamente para el análisis de la COVID-19 titulado 
“The vulnerability of functions" publicado en Academia Letters. Y resulta realmente atendible... Como éste hay múltiples ejemplos, aunque hay que tener cuidado con aquello que advierten David Alexander y JC Gaillard: el Golden rush, la fiebre del oro.

Sin duda los avances que al momento tenemos dentro de lo que genéricamente podemos denominar el estudio social e histórico de los desastres ha dejado una cantidad muy apreciable de aprendizajes. Ello ha permitido hoy comprender mejor, analizar con mayor precisión y mostrar elementos que son útiles para las personas en riesgo, pero también para los responsables de la toma de decisiones con el fin de gestionar los desastres de manera más oportuna y eficaz. Ello fue producto de un largo viaje, a lo largo del cual buscábamos identificar y reconocer las causas originales responsables de que los eventos extremos de la naturaleza se conviertan en desastres. El itinerario de ese viaje incluyó varias rutas, entre ellas: combinar diferentes perspectivas; explorar en regiones variadas y contextos diversos; estudiar casos específicos, tanto históricos como contemporáneos, de desastres asociados con amenazas naturales y biológicas.

\section{La Pandemia COVID-19}

No obstante, y a pesar de todo lo anterior, con la experiencia derivada de la pandemia asociada al COVID-19 pareciera que estamos comenzando un nuevo viaje, soslayando y omitiendo quizás por ignorancia o por tozudez, los progresos alcanzados a través del análisis de desastres. Actualmente, con el surgimiento de esta pandemia, el enfoque de la medicina, específicamente la epidemiología, está empezando a prevalecer en todas las latitudes como un motor clave para la toma de decisiones. Al igual que en el pasado, los meteorólogos o geofísicos eran la voz cantante para decidir cómo actuar frente a eventos relacionados con el clima o con la presencia de terremotos que desencadenaron. Ello era producto, como mencioné antes, de atribuir la ocurrencia de los desastres de manera directa a la presencia de determinados fenómenos naturales, de donde derivó el término "desastre natural", el cual se ha usado y del cual se ha abusado particularmente en las últimas décadas.

Al lado de los científicos naturales están los científicos exactos. Se privilegia la información de índole cuantitativo por encima de la de orden cualitativo. El 80\% de los datos que escuchamos, que leemos, que se nos presentan constantemente a través de los medios son numéricos: suma de confinados, total de sospechosos, mortalidad vs letalidad, monitoreos. Excelentes, sin duda, pero que constituyen indicadores para profundizar en los muchos temas que competen a las ciencias sociales y que permiten entender el porqué y el cómo de esas cifras. Y, por supuesto, la asociación de esa numeralia con las medidas diferenciales adoptadas por país o incluso por región dentro de un mismo país: los costos sociales, por decir lo menos, de ese encierro mundial. 
Recientemente algunos miembros de LA RED aludieron a esta discusión, en un documento cuyo objetivo es examinar lo que ha ocurrido con la COVID-19 (y diga "la COVID" porque se refiere a la enfermedad, de ahí que esté en femenino), justamente no desde la perspectiva de la epidemiología, sino considerándolo un desastre que expresa el riesgo subyacente. Y lo hacen precisamente a la luz de los conocimientos y experiencias acumuladas en varias décadas de estudios sociales interdisciplinarios sobre el tema (LAVELL et al. 2020). Utilizan conceptos desarrollados con esa perspectiva para interpretar la construcción social del riesgo para el virus en asociación con la vida humana, los sistemas de salud pública y la economía. Argumentan que, como en otros desastres, confundir el riesgo con el virus invisibiliza esos otros factores de riesgo subyacentes, mientras que las políticas de gestión, por su parte, pueden conducir a una transferencia de riesgo a quienes resultan ser, como suele suceder en casos de desastre, los más expuestos y vulnerables en este caso al coronavirus.

Parte de ello analizaremos en dos publicaciones que aparecerán (esperamos) a lo largo de 2022 por Berghahn Books:

- Con Susanna M. Hoffman, Inplacement: Global Outbreaks and the Anthropology of Isolation (Anthropology and COVID-19);

- Con Roberto E. Barrios, A Revelatory Pandemic? Disaster, Social Science and COVID 19 in Latin America.

Sin duda de la experiencia de esta pandemia global, que no es la primera ni será la última, tenemos mucho que aprender dentro del campo de los desastres, de su desnaturalización, de las resistencias surgidas y de las movilizaciones en proceso y las que están por venir. 


\section{Referencias}

GARCÍA ACOSTA, Virginia. "Vulnerabilidad y desastres. Génesis y alcances de una visión alternativa", en: Mercedes González de la Rocha y Gonzalo A. Saraví (coords.), Pobreza y vulnerabilidad: debates y estudios contemporáneos en México, Centro de Investigaciones y Estudios Superiores en Antropología Social, México, pp. 212-239, 2018.

HEWITT, Kenneth (ed.). Interpretations of Calamity from the Viewpoint of Human Ecology. Allen and Unwin, Londres, 1983.

LAVELL, Allan. Los conceptos, estudios y práctica en torno al tema de los riesgos y desastres en América Latina: evolución y cambio, 1980-2004. El rol de LA RED, sus miembros y sus instituciones de apoyo, Consejo Latinoamericana de Ciencias Sociales, San José, 2005. Disponible en línea: <http://biblioteca.clacso.edu.ar/Argentina/flacso-ar/20190801044010/lavell.pdf>. Consultado el 6 de junio de 2020.

LAVELL, Allan; MANSILLA, Elizabeth; MASKREY, Andrew; RAMÍREZ, Fernando. The Social Construction of the Covid-19 Pandemic: Disaster, Risk Accumulation and Public Policy, en LA RED, 21 de Agosto de 2020. Disponible en línea: <https://www. desenredando.org/>. Consultado el 20 de mayo de 2020.

MASKREY, Andrew (comp.). Los desastres no son naturales. LA RED, Lima, 1993.

O'KEEFE, Phil, WESTGATE, Ken; WISNER, Ben . Taking the Naturalness Out of Natural Disasters, en Nature, n. 260, p. 566-567, 1976.

REVET, Sandrine. Disasterland: An Ethnography of the International Disaster Community, Palgrave MacMillan (Sciences Po Series in International Relations and Political Economy), Cham, 2020. 